\title{
GENERALIZED DEFORMED OSCILLATOR FOR VORTICES IN SUPERFLUID FILMS
}

\author{
DENNIS BONATSOS \\ Institute of Nuclear Physics, NCSR "Demokritos" \\ GR-15310 Aghia Paraskevi, Attiki, Greece \\ C. DASKALOYANNIS \\ Department of Physics, Aristotle University of Thessaloniki \\ GR-54006 Thessaloniki, Greece
}

\begin{abstract}
The algebra of observables of a system of two identical vortices in a superfluid thin film is described as a generalized deformed oscillator with a structure function containing a linear (harmonic oscillator) term and a quadratic term. In contrast to the deformed oscillators occuring in other physical systems (correlated fermion pairs in a single- $j$ nuclear shell, Morse oscillator), this oscillator is not amenable to perturbative treatment and cannot be approximated by quons. From the mathematical viewpoint, this oscillator provides a novel boson realization of the algebra $\mathrm{su}(1,1)$.
\end{abstract}

\footnotetext{
${ }^{1}$ Also at the European Centre for Theoretical Studies in Nuclear Physics and Related Areas (ECT*), Strada delle Tabarelle 286, I-38050 Villazzano (Trento), Italy. E-mail: bonat@ectstar.ect.unitn.it, bonat@cyclades.nrcps.ariadne-t.gr

${ }^{2}$ E-mail: daskaloyanni@olymp.ccf.auth.gr
} 
The possibility of the existence in two dimensions of statistics intermediate between the bosonic and fermionic ones has attracted recently much attention ${ }^{1}$, since it is related to questions concerning the fractional quantum Hall effect and anyon superconductivity. The quantization of the system of two identical vortices has also attracted much attention, since it has been suggested as an anyon candidate ${ }^{2-7}$, although opposing views also exist ${ }^{8}$.

On the other hand, quantum algebras (quantum groups) ${ }^{9,10}$ which are nonlinear generalizations of the usual Lie algebras to which they reduce for appropriate values of the deformation parameter(s), have been finding applications as the dynamical symmetry algebras of several physical systems. For the boson realization of these algebras, various kinds of deformed oscillators have been introduced ${ }^{11-15}$ and unification schemes for them have been suggested (see ${ }^{16}$ for a list of references). In the framework of intermediate statistics the term quons has been used for some of these oscillators ${ }^{17,18}$.

In the present work the quantization of a system of two identical vortices in a superfluid thin film will be considered. It will be demonstrated that the algebra of the observables of this system is a generalized deformed oscillator 16,19 with a structure function containing a linear term, which corresponds to the usual harmonic oscillator, and a quadratic term. This oscillator is not amenable to perturbative treatment and cannot be approximated by quons, which do provide an adequate description of other physical systems.

Let us consider a system of two identical massless point vortices in an unbounded flat, frictionless, incompressible, superfluid thin film ${ }^{2,7}$. If the vortices are centered at the points $\left(x_{i}, y_{i}\right), i=1,2$, we introduce the relative coordinates

$$
x=x_{1}-x_{2}, \quad y=y_{1}-y_{2},
$$

as well as the center of vorticity coordinates

$$
X=\frac{x_{1}+x_{2}}{2}, \quad Y=\frac{y_{1}+y_{2}}{2} .
$$

The center of vorticity coordinates are constants of motion, while the equations of motion for the relative coordinates are

$$
\kappa \frac{d x}{d t}=2 \frac{\partial H}{\partial y}, \quad \kappa \frac{d y}{d t}=-2 \frac{\partial H}{\partial x}
$$


where $\kappa$ is the quantized vorticity of the superfluid ${ }^{7}$, given by

$$
\kappa=\frac{2 \pi \hbar}{m}
$$

where $m$ is the mass of the atoms in the superfluid. We will assume that $\kappa$ is positive. (Negative $\kappa$ corresponds to vortices with opposite circulation.) The relevant Hamiltonian is ${ }^{6}$

$$
H=-\frac{\kappa^{2}}{4 \pi} \ln \left(\frac{x^{2}+y^{2}}{a^{2}}\right),
$$

where $a$ is a scale parameter. The energy $E$ of the system is related to the Hamiltonian $H$ by

$$
E=\rho \delta H,
$$

where $\rho$ is the density and $\delta$ the thickness of the superfluid film. The momentum conjugate to $x$, with appropriate dimensions, is

$$
p_{x}=\frac{\kappa \rho \delta}{2} y
$$

the canonical quantization condition being

$$
\left[x, p_{x}\right]=i \hbar, \quad \text { or } \quad[x, y]=\frac{2 i \hbar}{\kappa \rho \delta} .
$$

The relative coordinates $x$ and $y$ are not invariant under the interchange of the position of the two vortices

$$
(x, y) \rightarrow(-x,-y)
$$

while the observables of the system have to be invariant under this interchange. Therefore quantities acceptable as observables are ${ }^{6}$

$$
\begin{aligned}
A & =\frac{1}{8} \kappa \rho \delta\left(x^{2}+y^{2}\right), \\
B & =\frac{1}{8} \kappa \rho \delta\left(y^{2}-x^{2}\right), \\
C & =\frac{1}{8} \kappa \rho \delta(x y+y x),
\end{aligned}
$$

satisfying the commutation relations

$$
[A, B]=i \hbar C, \quad[A, C]=-i \hbar B, \quad[B, C]=-i \hbar A
$$


Introducing the operators

$$
B_{ \pm}=B \pm i C
$$

the commutation relations take the form ${ }^{6}$

$$
\left[A, B_{ \pm}\right]= \pm \hbar B_{ \pm}, \quad\left[B_{+}, B_{-}\right]=-2 \hbar A
$$

Defining

$$
\tilde{A}=\frac{A}{\hbar}, \quad \tilde{B}_{ \pm}= \pm \frac{B_{ \pm}}{\hbar},
$$

one sees that these commutation relations acquire the $\mathrm{su}(1,1)$ form

$$
\left[\tilde{A}, \tilde{B}_{ \pm}\right]= \pm \tilde{B}_{ \pm}, \quad\left[\tilde{B}_{+}, \tilde{B}_{-}\right]=-2 \tilde{A}
$$

For later use we also remark that the first of these equations can be written as

$$
\left[\frac{A}{\hbar}, B_{ \pm}\right]= \pm B_{ \pm}
$$

On the other hand, it is known that a deformed oscillator ${ }^{16,19}$ can be defined by the algebra generated by the operators $\left\{1, a, a^{\dagger}, N\right\}$ and the structure function $\Phi(x)$, satisfying the relations:

$$
[a, N]=a, \quad\left[a^{\dagger}, N\right]=-a^{\dagger}
$$

and

$$
a^{\dagger} a=\Phi(N)=[N], \quad a a^{\dagger}=\Phi(N+1)=[N+1],
$$

where $\Phi(x)$ is a positive analytic function with $\Phi(0)=0$ and $N$ is the number operator. From Eq. (18) we conclude that:

$$
N=\Phi^{-1}\left(a^{\dagger} a\right)
$$

and that the following commutation and anticommutation relations are obviously satisfied:

$$
\left[a, a^{\dagger}\right]=[N+1]-[N], \quad\left\{a, a^{\dagger}\right\}=[N+1]+[N] .
$$

The structure function $\Phi(x)$ is characteristic to the deformation scheme. In Table 1 the structure functions corresponding to various deformed oscillators are given. 
Generalized deformed algebras possess a Fock space of eigenvectors $\mid 0>$, $|1>, \ldots| n>,, \ldots$ of the number operator $N$

$$
N|n>=n| n>, \quad<n \mid m>=\delta_{n m},
$$

if the vacuum state $\mid 0>$ satisfies the following relation:

$$
a \mid 0>=0 .
$$

These eigenvectors are generated by the formula:

$$
\left|n>=\frac{1}{\sqrt{[n] !}}\left(a^{\dagger}\right)^{n}\right| 0>,
$$

where

$$
[n] !=\prod_{k=1}^{n}[k]=\prod_{k=1}^{n} \Phi(k) .
$$

The generators $a^{\dagger}$ and $a$ are the creation and destruction operators of this deformed oscillator algebra:

$$
a|n>=\sqrt{[n]}| n-1>, \quad a^{\dagger}|n>=\sqrt{[n+1]}| n+1>.
$$

Returning to the system of two vortices, we see that the first equation in Eq. (15) has the same form as Eq. (17), with $B_{+}\left(B_{-}\right)$playing the role of the creation (annihilation) operator, and $A$ being related to the number operator

$$
B_{+}=a^{\dagger}, \quad B_{-}=a, \quad \frac{A}{\hbar}=N+u,
$$

where $u$ is a constant to be determined later.

Performing a straightforward calculation one can show that

$$
B_{+} B_{-}=A^{2}-\hbar A+\frac{3}{16} \hbar^{2}
$$

Substituting in Eq. (27) $A$ from Eq. (26) one obtains

$$
B_{+} B_{-}=\Phi(N), \quad B_{-} B_{+}=\Phi(N+1),
$$

where $\Phi(x)$ is the structure function given by

$$
\Phi(x)=\hbar^{2}\left((x+u)^{2}-(x+u)+\frac{3}{16}\right) .
$$


As already mentioned, the structure function has to satisfy the conditions

$$
\Phi(0)=0, \quad \Phi(x) \geq 0 \quad \text { for } \quad x \geq 0,
$$

which imply

$$
u=\frac{3}{4},
$$

and therefore

$$
\Phi(x)=\hbar^{2}\left(x^{2}+\frac{x}{2}\right),
$$

while from Eq. (26) one has

$$
A=\hbar\left(N+\frac{3}{4}\right), \quad N=0,1,2, \ldots
$$

The basis in the present case is given by

$$
\left|n>=\frac{1}{\sqrt{[n] !}}\left(B_{+}\right)^{n}\right| 0>,
$$

where

$$
[n] !=\prod_{k=1}^{n} \Phi(k) .
$$

In this basis one has

$$
N|n>=n| n>,
$$

the eigenvalues of the operator $A$ being

$$
A_{n}=\hbar\left(n+\frac{3}{4}\right) .
$$

Since the Hamiltonian (Eq. 5) can be written as

$$
H=-\frac{\kappa^{2}}{4 \pi} \ln \left(\frac{8}{\kappa \rho \delta a^{2}} A\right),
$$

the energy eigenvalues are

$$
E_{n}=-\frac{\rho \delta \kappa^{2}}{4 \pi} \ln \left(\frac{8 \hbar}{\kappa \rho \delta a^{2}}\left(n+\frac{3}{4}\right)\right),
$$

in agreement with Eq. (17) of ${ }^{6}$.

We have therefore constructed a deformed oscillator describing the system of two vortices. The following comments are now in place: 
i) As seen in Table 1, the structure function for usual bosons (harmonic oscillator) is $\Phi(N)=N$, while in the present case of the system of two vortices one has $\Phi(N)=\hbar^{2}\left(N / 2+N^{2}\right)$, i.e. the structure function is a linear combination of a bosonic and a quadratic term, indicating the relation of the two-vortex system to intermediate statistics ${ }^{1-5}$.

ii) The algebra of observables studied here is the same as the one occuring for a system of two anyons in a strong magnetic field ${ }^{29}$.

iii) The assumption that vortices are point-like is known to be quite restrictive ${ }^{28}$.

iv) Deformed oscillators have been found useful in describing some other physical systems. In the case of correlated fermion pairs of zero angular momentum in a single- $j$ nuclear shell the structure function of the relevant oscillator is ${ }^{30}$

$$
\Phi(N)=\left(1+\frac{1}{\Omega}\right) N-\frac{N^{2}}{\Omega},
$$

where $2 \Omega=2 j+1$ is the size of the shell, while in the case of the Morse oscillator the structure function has the form ${ }^{31}$

$$
\Phi(N)=N-x_{e} N^{2},
$$

where $x_{e}$ is the anharmonicity constant. Both cases can be viewed as perturbed harmonic oscillators, since the parameters $1 / \Omega$ and $x_{e}$ are small. In the case of the two-vortex system, however, no small parameter appears, the $N^{2}$ term having a coefficient twice as large as the one of $N$. Therefore no perturbative treatment is possible in this case.

v) The deformed bosons introduced in the two examples mentioned above (single- $j$ shell, Morse potential), can be approximated by $Q$-bosons ${ }^{11,12}$, characterized by the structure function

$$
F(N)=\frac{Q^{N}-1}{Q-1},
$$

where $Q=e^{T}$ (with $T$ real). In the former case the deformation parameter $T$ is related to the size of the shell by $T=-2 / \Omega^{32}$, while in the latter it is connected to the anharmonicity constant by $T=-2 x_{e}{ }^{31}$. In the present case of the two-vortex system the deformed bosons cannot be approximated by 
$Q$-bosons, due to the lack of a small parameter. $Q$-bosons are also appearing in the literature of intermediate statistics under the name of quons ${ }^{17,18}$.

vi) It should always be kept in mind that in the cases of the two examples mentioned above the energy of the system is given by the energy of the oscillator, while in the case of the two vortices the energy of the system is related to the number operator of the deformed oscillator.

vii) The deformed oscillator operators $B_{+}, B_{-}$and $N$ provide a new boson mapping for the generators of the $\mathrm{su}(1,1)$ algebra of Eq. (15), through

$$
\tilde{B}_{+}=\frac{a^{\dagger}}{\hbar}, \quad \tilde{B}_{-}=\frac{a}{\hbar}, \quad \tilde{A}=N+\frac{3}{4} .
$$

Existing boson mappings ${ }^{33-35}$ of $\mathrm{su}(1,1)$ in terms of usual bosons include the Schwinger realization

$$
\tilde{B}_{+}=a_{1}^{\dagger} a_{2}^{\dagger}, \quad \tilde{B}_{-}=a_{1} a_{2}, \quad \tilde{A}=\frac{1}{2}\left(N_{1}+N_{2}+1\right),
$$

where

$$
N_{1}=a_{1}^{\dagger} a_{1}, \quad N_{2}=a_{2}^{\dagger} a_{2},
$$

which involves two sets of bosons, $\left(a_{1}^{\dagger}, a_{1}, N_{1}\right.$ and $\left.a_{2}^{\dagger}, a_{2}, N_{2}\right)$, as well as the realization

$$
\tilde{B}_{+}=\frac{1}{2}\left(a^{\dagger}\right)^{2}, \quad \tilde{B}_{-}=\frac{1}{2} a^{2}, \quad \tilde{A}=\frac{1}{2}\left(N+\frac{1}{2}\right),
$$

with

$$
N=a^{\dagger} a
$$

involving one boson. In both of these realizations the $\mathrm{su}(1,1)$ operators are mapped onto bilinear combinations of bosons, while in the present case they are mapped onto terms linear in the deformed bosons.

viii) An expression similar to Eq. (39) for the energy of a pair of interacting massless vortices in two dimensions has been derived in ${ }^{36}$, where different quantization schemes have been compared. This energy is given by

$$
E_{N}=-\frac{1}{4} \quad q^{2} \quad \frac{n_{1} n_{2}}{2 \pi} \quad \ln \left(\frac{2 c}{r_{0}^{2}} \quad(2 N+1)\right),
$$

where $q^{2}=\rho_{0} \sigma^{2}, \rho_{0}$ is the fluid mass-density, $\sigma=h / m$ is the vorticity (same as $\kappa$ of Eq. (4) in our notation), $r_{0}$ is the vortex core size, and $n_{i}$ are integers describing the vorticity quantization ${ }^{2,36,37}$. 
ix) A canonical quantization scheme for vortices in superfluid He II has been given in ${ }^{38,39}$. An infinite Lie algebra of incompressible flows is obtained, introduced by the quantization in the theory.

In conclusion, we have shown that the algebra of observables of a system of two identical vortices in a superfluid thin film can be described in terms of a generalized deformed oscillator characterized by a structure function containing a linear (harmonic oscillator) term and a quadratic term. From the mathematical point of view, this deformed oscillator provides a novel boson realization of the $\mathrm{su}(1,1)$ algebra. In contrast to other physical systems (correlated fermion pairs, Morse oscillator) the two-vortex system cannot be seen as a perturbed harmonic oscillator and cannot be approximated by $Q-$ bosons or quons.

\section{Acknowledgment}

Support by the Greek Secretariat of Research and Technology under contract PENED95/1981 and by CEC under contract ERBCHBGCT930467 (DB) is gratefully acknowledged. 


\section{References}

1. J. M. Leinaas, Phys. Reports 242, 371 (1994).

2. R. Y. Chiao, A. Hansen and A. A. Moulthrop, Phys. Rev. Lett. 54, 1339 (1985).

3. A. Hansen, R. Y. Chiao and A. A. Moulthrop, Phys. Rev. Lett. 55, 1431 (1985).

4. F. D. M. Haldane and Y. S. Wu, Phys. Rev. Lett. 55, 2887 (1985).

5. G. A. Goldin, R. Menikoff and D. H. Sharp, Phys. Rev. Lett. 58, 174 (1987).

6. J. M. Leinaas and J. Myrheim, Phys. Rev. B 37, 9286 (1988).

7. L. Onsager, Nuovo Cimento 6, Suppl., 279 (1979).

8. J. Dziarmaga, cond-mat/9504004.

9. V. G. Drinfeld, in Proceedings of the International Congress of Mathematicians, ed. A. M. Gleason (American Mathematical Society, Providence, RI, 1986), p. 798.

10. M. Jimbo, Lett. Math. Phys. 11, 247 (1986).

11. M. Arik and D. D. Coon, J. Math. Phys. 17, 524 (1976).

12. V. V. Kuryshkin, Annales de la Fondation Louis de Broglie 5, 111 (1980).

13. L. C. Biedenharn, J. Phys. A 22, L873 (1989).

14. A. J. Macfarlane, J. Phys. A 22, 4581 (1989).

15. C. P. Sun and H. C. Fu, J. Phys. A 22, L983 (1989).

16. D. Bonatsos and C. Daskaloyannis, Phys. Lett. B 307, 100 (1993).

17. O. W. Greenberg, U. Maryland preprint 93-097 (1993).

18. S. Meljanac and A. Perica, J. Phys. A 27, 4737 (1994).

19. C. Daskaloyannis, J. Phys. A 24, L789 (1991).

20. R. Chakrabarti and R. Jagannathan, J. Phys. A 24, L711 (1991).

21. Y. Ohnuki and S. Kamefuchi, Quantum Field Theory and Parastatistics (Springer-Verlag, Berlin, 1982).

22. K. Odaka, T. Kishi and S. Kamefuchi, J. Phys. A 24, L591 (1991).

23. A. Jannussis, G. Brodimas, D. Sourlas and V. Zisis, Lett. Nuovo Cimento 30, 123 (1981).

24. T. Hayashi, Commun. Math. Phys. 127, 129 (1990).

25. D. Bonatsos and C. Daskaloyannis, J. Phys. A 26, 1589 (1993). 
26. K. S. Viswanathan, R. Parthasarathy and R. Jagannathan, J. Phys. A 25, L335 (1992).

27. R. Chakrabarti and R. Jagannathan, J. Phys. A 27, L277 (1994).

28. G. A. Goldin, R. Menikoff and D. H. Sharp, Phys. Rev. Lett. 58, 2162 (1987).

29. T. H. Hansson, J. M. Leinaas and J. Myrheim, Nucl. Phys. B 384, 559 (1992).

30. D. Bonatsos and C. Daskaloyannis, Phys. Lett. B 278, 1 (1992).

31. D. Bonatsos and C. Daskaloyannis, Chem. Phys. Lett. 203, 150 (1993).

32. D. Bonatsos, J. Phys. A 25, L101 (1992).

33. A. Klein and E. R. Marshalek, Rev. Mod. Phys. 63, 375 (1991).

34. V. Penna, Mod. Phys. Lett. B 5, 1947 (1991).

35. V. Barone, V. Penna and P. Sodano, Phys. Lett. A 161, 41 (1991).

36. V. Penna, Phys. Lett. A 125, 385 (1987).

37. V. Penna, Physica A 152, 400 (1988).

38. M. Rasetti and T. Regge, Physica A 80, 217 (1975).

39. M. Rasetti and T. Regge, Quantum Vortices, in Highlights of Condensed Matter Theory, ed. F. Bassani et al. (Compositori, Bologna, 1985). 
Table 1: Structure functions of special deformation schemes

\begin{tabular}{|c|c|c|}
\hline & $\Phi(x)$ & Reference \\
\hline$\overline{i \mathrm{i}}$ & $\bar{x}$ & harmonic oscillator \\
\hline ii & $\frac{q^{x}-q^{-x}}{q-q^{-1}}=[x]_{q}$ & $\begin{array}{l}q \text {-deformed harmonic oscil- } \\
\text { lator } 13-15\end{array}$ \\
\hline iii & $\frac{Q^{x}-1}{Q-1}=[x]_{Q}$ & $Q$-deformed oscillator ${ }^{11,12}$ \\
\hline iv & $\frac{q^{x}-p^{-x}}{q-p^{-1}}=[x]_{p, q}$ & $\begin{array}{l}(p, q) \text {-deformed } \\
\text { 2-parameter oscillator } 20\end{array}$ \\
\hline $\mathrm{v}$ & $x(p+1-x)$ & parafermionic oscillator ${ }^{21}$ \\
\hline vi & $\frac{\sinh (\tau x) \sinh (\tau(p+1-x))}{\sinh ^{2}(\tau)}$ & $\begin{array}{l}q \text {-deformed parafermionic } \\
\text { oscillator } 22\end{array}$ \\
\hline vii & $x \cos ^{2}(\pi x / 2)+(x+p-1) \sin ^{2}(\pi x / 2)$ & parabosonic oscillator ${ }^{21}$ \\
\hline viii & $\begin{array}{l}\frac{\sinh (\tau x)}{\sinh (\tau)} \frac{\cosh \left(\tau\left(x+2 N_{0}-1\right)\right)}{\cosh (\tau)} \cos ^{2}(\pi x / 2)+ \\
+\frac{\sinh \left(\tau\left(x+2 N_{0}-1\right)\right)}{\sinh (\tau)} \frac{\cosh (\tau x)}{\cosh (\tau)} \sin ^{2}(\pi x / 2)\end{array}$ & $\begin{array}{l}q \text {-deformed parabosonic os- } \\
\text { cillator } 22\end{array}$ \\
\hline ix & $\sin ^{2} \pi x / 2$ & fermionic algebra ${ }^{23}$ \\
\hline $\mathrm{x}$ & $q^{x-1} \sin ^{2} \pi x / 2$ & $\begin{array}{l}q \text {-deformed fermionic alge- } \\
\text { bra } 24\end{array}$ \\
\hline $\mathrm{xi}$ & $x(2-x)$ & fermionic algebra ${ }^{21}$ \\
\hline xii & $\frac{\sin (\tau x) \sin (\tau(2-x))}{\sin ^{2}(\tau)}$ & $\begin{array}{l}q \text {-deformed fermionic alge- } \\
\text { bra } 22,25\end{array}$ \\
\hline xiii & $\frac{1-(-q)^{x}}{1+q}$ & $\begin{array}{l}\text { generalized } q \text {-deformed } \\
\text { fermionic algebra } 26\end{array}$ \\
\hline xiv & {$[x]_{p, q}+2 \nu[x]_{-p, q}$} & modified $(p, q)$-oscillator ${ }^{27}$ \\
\hline $\mathrm{xv}$ & $x^{n}$ & 19 \\
\hline xvi & $\frac{s n(\tau x)}{s n(\tau)}$ & 19 \\
\hline
\end{tabular}

